\title{
Multiple cerebral infarction and paradoxical air embolism during hepatectomy using the Cavitron Ultrasonic Surgical Aspirator
} -A case report-

\author{
Jae Hoon Lee, Tae Dong Kwon, Hyun Joo Kim, Byoungchan Kang, and Bon-Nyeo Koo \\ Department of Anesthesiology and Pain Medicine, Anesthesia \& Pain Research Institute, Yonsei University College of Medicine, \\ Seoul, Korea
}

A venous air embolism and paradoxical air embolism (PAE) are serious complications in patients undergoing a hepatectomy. We report a case of PAE and cerebral infarctions in a patient undergoing a hepatic resection using a Cavitron Ultrasonic Surgical Aspirator $\left(\right.$ CUSA $\left.^{\circledR}\right)$. A 65-year-old woman underwent a left lobe hepatectomy. During the middle phase of the liver resection with CUSA ${ }^{\circledR}$, there was a sudden decrease in arterial blood pressure, end-tidal carbon dioxide and $\mathrm{SpO}_{2}$. With resuscitation, intraoperative ultrasonography revealed massive air emboli in both her left and right heart, which lasted for $40 \mathrm{~min}$. The hepatectomy was completed after the disappearance of the air emboli from her heart. After surgery, her mental status was stuporous. The brain CT and MRI revealed multiple acute cerebral infarctions. Finally, she died from septic shock. This case highlights the need for anesthetists and surgeons to be aware of the potential for CUSA ${ }^{\circledR}$-related massive PAE. (Korean J Anesthesiol 2010; 59: S133-S136)

Key Words: Hepatectomy, Liver disease, Paradoxical embolism.

In 2005, Koo et al. [1] reported that all patients who underwent a liver resection using the Cavitron Ultrasonic Aspirator (CUSA ${ }^{\circledR}$ ) presented with a venous air embolism (VAE), a potentially fatal complication. One year later, Adachi et al. [2] reported that within 6 months of introducing the CUSA ${ }^{\circledR}$ device in their University Hospital, they had encountered 3 cases of cardiac arrest during a hepatic resection. Their patients were diagnosed with VAE, and CUSA ${ }^{\circledR}$-related air entrainment from the liver was strongly suspected. Systemic VAE is a serious complication in patients undergoing a hepatectomy. Arteriovenous (AV) shunting or massive air emboli in a patient can pass into the systemic circulation (paradoxical air embolism, PAE). We report a case of PAE and multiple cerebral infarctions in a patient with cirrhosis, who underwent a hepatic resection using CUSA ${ }^{\circledR}$.

Received: May 28, 2010. Revised: 1st, June 11, 2010; 2nd, July 19, 2010. Accepted: August 6, 2010.

Corresponding author: Bon-Nyeo Koo, M.D., Ph.D., Department of Anesthesiology and Pain Medicine, Anesthesia \& Pain Research Institute, Yonsei University College of Medicine, 134, Shinchon-dong, Seodaemun-gu, Seoul 120-752, Korea. Tel: 82-2-2228-2420, Fax: 82-2-312-7185, E-mail:koobn@yuhs.ac

(c) This is an open-access article distributed under the terms of the Creative Commons Attribution Non-Commercial License (http:// creativecommons.org/licenses/by-nc/3.0/), which permits unrestricted non-commercial use, distribution, and reproduction in any medium, provided the original work is properly cited. 


\section{Case Report}

A 65-year-old woman was scheduled to undergo a left lobe hepatectomy due to a hepatocellular carcinoma. She had no medical problems with the only exception being liver cirrhosis (Child-Pugh class A) due to chronic hepatitis B. After administering intravenous glycoppyrolate $0.2 \mathrm{mg}$ as premedication, general anesthesia was induced with propofol $100 \mathrm{mg}$, rocuronium $50 \mathrm{mg}$ and remifentanil $40 \mu \mathrm{g}$, and maintained with sevoflurane $(1-2.5 \%)$, remifentanil $(0.1-$ $0.25 \mu \mathrm{g} / \mathrm{kg} / \mathrm{min}$ ) and rocuronium (5-10 $\mu \mathrm{g} / \mathrm{kg} / \mathrm{min})$. She was intubated and ventilated mechanically using an anesthesia machine, and monitored continuously by electrocardiography (EKG), arterial blood pressure, central venous pressure and $\mathrm{SpO}_{2}$.

Her vital signs were stable until the middle phase of the liver resection. One hour after starting the hepatectomy with CUSA $^{\circledR}$, there was a sudden decrease in arterial blood pressure (systolic blood pressure $<40 \mathrm{mmHg}$ ), end-tidal carbon dioxide $(<26 \mathrm{mmHg})$ and $\mathrm{SpO}_{2}(<50 \%)$. She also showed tachycardia (110-135 beats/min) and a ST elevation on the EKG. Upon resuscitation with the rapid intravenous administration of colloid and catecholamines (intravenous epinephrine $1 \mathrm{mg}$, infusion of norepinephrine), intraoperative ultrasonography revealed massive air emboli in both her left and right heart (Fig. 1), and she was diagnosed with VAE and PAE. At diagnosis, arterial blood gas analysis (ABGA) showed the following: $\mathrm{pH}$ (7.278), $\mathrm{pCO}_{2}$ (51.3 mmHg), $\mathrm{PO}_{2}(84.6 \mathrm{mmHg}) \mathrm{HCO}_{3}{ }^{-}(24.2$ mmHg), $\mathrm{SaO}_{2}(94.4 \%)$ on $\mathrm{FiO}_{2}(0.5)$. From 10 minutes after catecholamine administration, her systolic blood pressure and

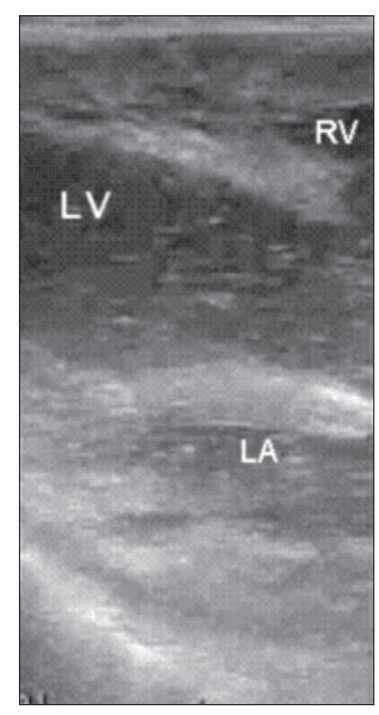

Fig. 1. Intraoperative sonogram showing the paradoxical air embolism. $\mathrm{LV}, \mathrm{RV}$ and LA present left ventricle, right ventricle and left atrium, respectively. heart rate were maintained at approximately $90 \mathrm{mmHg}$ and 110 beats/min. Her central venous pressure was approximately $2 \mathrm{mmHg}$, which is similar to that before the occurrence of VAE. End-tidal carbon dioxide was restored to $32 \mathrm{mmHg}$. ABGA at 30 minutes after the occurrence of this episode revealed the following: $\mathrm{pH}$ (7.338), $\mathrm{pCO}_{2}$ (44.3 $\mathrm{mmHg}$ ), $\mathrm{PO}_{2}$ (234.0 mmHg), $\mathrm{HCO}_{3}{ }^{-}$(24.0 mmHg), $\mathrm{SaO}_{2}$ (99.6\%) on $\mathrm{FiO}_{2}$ (1.0). Norepinephrine infusion $(0.2-0.3 \mu \mathrm{g} / \mathrm{kg} / \mathrm{min})$ and fluid resuscitation was continued (colloid $1000 \mathrm{ml}$ and crystalloid $500 \mathrm{ml}$ was infused) until the air in her heart had disappeared. The air emboli in her left heart disappeared 70 min later, even though an attempt was made to aspirate the air using an internal jugular venous catheter. The hepatectomy was restarted and completed after confirming the disappearance of air emboli from her heart by intraoperative ultrasonography. From the occurrence of VAE to the end of surgery, her systolic pressure was maintained at approximately $90 \mathrm{mmHg}$ despite the norepinephrine infusion $(0.2-0.3 \mu \mathrm{g} / \mathrm{min} / \mathrm{kg})$. During the operation (total anesthesia time: $5 \mathrm{hr}$ ), 2,950 $\mathrm{ml}$ of fluid had been administered. The total urinary output and blood loss was $220 \mathrm{ml}$ and $800 \mathrm{ml}$, respectively.

After surgery, in the intensive care unit, she was still intubated and ventilated mechanically. She responded only to intense pain. Her systolic pressure was still maintained at approximately $90 \mathrm{mmHg}$ despite the norepinephrine infusion $(0.2-0.3 \mu \mathrm{g} /$ $\mathrm{min} / \mathrm{kg}$ ). Her postoperative laboratory findings revealed an abnormal PT/PTT (25.5/51.8 sec), fibrinogen (96 mg/dl), d-dimer (6,519 ng/ml), antithrombin III (19\%), CK-MB (40.74 $\mathrm{ng} / \mathrm{ml})$ and troponin-T $(1.880 \mathrm{ng} / \mathrm{ml})$, and the postoperative EKG showed a ST elevation in II, III and aVF. At postoperative day (POD) 1, her EKG findings recovered normally and the trans-thoracic echocardiogram to evaluate her cardiac function showed unremarkable findings and did not reveal the existence of patent foramen ovale (PFO). Her vital signs were stable and the norepinephrine infusion was tapered out. However, at POD 5, her mental status had not changed. The brain CT and MRI revealed multiple acute cerebral infarctions (Fig. 2). At POD 11, despite the unchanged mental status, she was weaned to spontaneous ventilation with CPAP mode and extubated. From POD 15, her vital signs became unstable and the intravenous administration of catecholamines was started. Finally, panperitonitis was confirmed by the identification of gram $(+)$ cocci on the peritoneal culture. At POD 31, she expired due to a cardiac arrest caused by septic shock.

\section{Discussion}

The VAE, PAE and multiple cerebral infarctions that occurred during a hepatectomy using CUSA ${ }^{\circledR}$ are presented. Previously, Koo et al. [1] reported that VAE occurs frequently and massively 

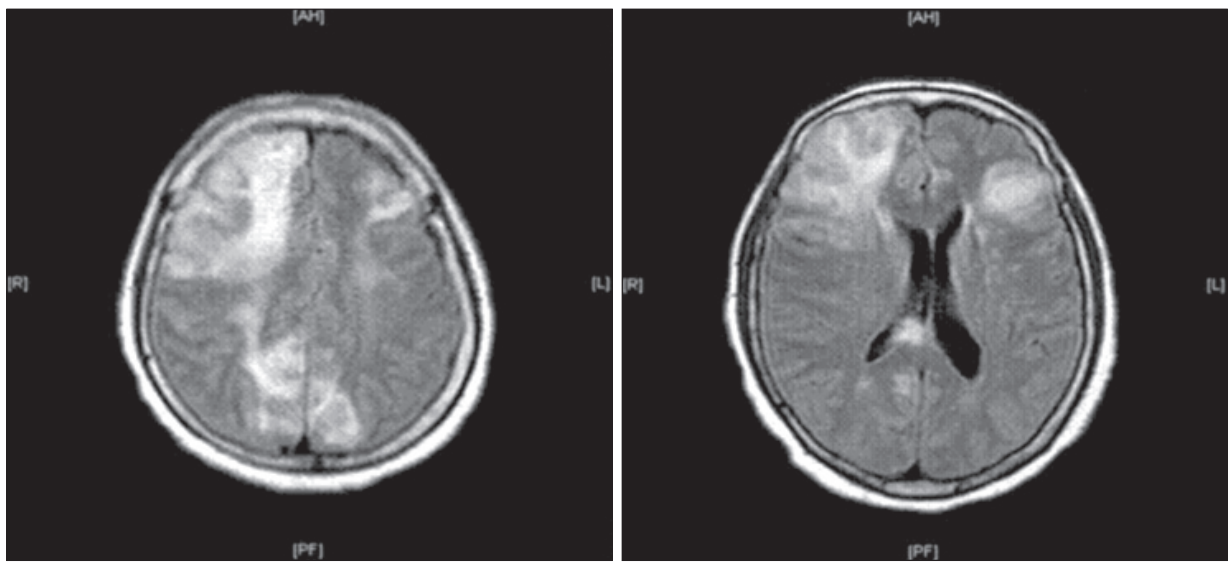

Fig. 2. Brain MRI at postoperative day 5 .

during a hepatectomy using CUSA ${ }^{\circledR}$. Although it was not believed to be clinically serious, previous studies strongly suggest that CUSA ${ }^{\circledR}$ related-PAE can develop and cause serious morbidity and mortality.

There is always the possibility that VAE can cause a pulmonary embolism and cardiopulmonary collapse. Furthermore, a paradoxical air embolism can cause major cerebral or coronary morbidity if there is an AV shunt or massive air emboli in the patient. A patent foramen ovale (PFO) serving as an AV shunt is present in approximately $25 \%$ of adults [3]. Although the precise pressure needed to open a PFO is not known, a clinical investigation showed that PAE occurs only in the context of major air embolic events, suggesting that significant increases in pressure in the right side of the heart are important predisposing factors for the occurrence of PAE [4]. In addition, air can occasionally traverse the pulmonary vascular bed to reach the systemic circulation $[5,6]$. Transpulmonary passage is more likely to occur when large volumes of air are presented to the pulmonary vascular filter [7].

There is another concern. It was previously reported that $15-45 \%$ of patients with liver cirrhosis have pulmonary abnormalities, including an intrapulmonary shunt caused by pulmonary vascular dilation and arteriovenous communication $[8,9]$. Therefore, paradoxical emboli can occur during an air embolism even if there are no intracardiac abnormalities present in patients with hepatic cirrhosis, such as PFO $[10,11]$. In our case, PFO could not be identified. However, the large entrained air volume may increase the pressure of the right side of the heart, which may open a tiny PFO. In addition, air may enter the left side of the heart through transpulmonary passage. The massive VAE in our case could have caused PAE. Finally, the patient suffered multiple cerebral infarctions as a result of PAE.

Although many studies reported that a hepatectomy using an ultrasonic aspirator is safe and simple [12-14], there is evidence suggesting that severe VAE and PAE can occur when using CUSA $^{\circledR}[1,2]$. Patients at risk of VAE or PAE should be monitored carefully for the immediate detection of cardiopulmonary collapse (continuous arterial pressure, $\mathrm{SpO}_{2}$, end tidal $\mathrm{CO}_{2}$ ) and CVP catheterization should be performed to aspirate the air in the right atrium. In particular, it is important that patients with limited cardiopulmonary reserve be monitored for the presence of VAE (the combination of precordial Doppler and expired $\mathrm{CO}_{2}$ monitoring, transesophageal echocardiography). In the present case, the omission of nitrous oxide, central venous catheterization, aspiration of entrained air and saline soaking of the surgical field were performed as recommended [1]. However, PAE and subsequent cerebral infarction could not be prevented. VAE was not diagnosed earlier because there were no significant signs until the massive VAE (systolic BP $<40$ $\mathrm{mmHg}, \mathrm{SaO}_{2}<50 \%$ ) had developed.

This case highlights the need for anesthetists and surgeons to be constantly aware of the possibility of CUSA ${ }^{\circledR}$ relatedmassive VAE and PAE. In addition, CUSA ${ }^{\circledR}$ should be applied for the minimum time possible and be practiced carefully. In addition, precautions should be taken to treat harmful VAE, particularly in those patients with liver cirrhosis. Furthermore, a brain ischemic injury should be the initial concern if PAE is confirmed.

\section{References}

1. Koo BN, Kil HK, Choi JS, Kim JY, Chun DH, Hong YW. Hepatic resection by the cavitron ultrasonic surgical aspirator increases the incidence and severity of venous air embolism. Anesth Analg 2005; 101: 966-70.

2. Adachi YU, Doi M, Sato S. Cardiac arrest by venous air embolism during hepatic resection using the cavitron ultrasonic surgical aspirator. Anesth Analg 2006; 103: 493-4.

3. Hagen PT, Scholz DG, Edwards WD. Incidence and size of patent foramen ovale during the first 10 decades of life: an autopsy study of 965 normal hearts. Mayo Clin Proc 1984; 59: 17-20.

4. Mammoto T, Hayashi Y, Ohnishi Y, Kuro M. Incidence of venous and paradoxical air embolism in neurosurgical patients in the 
sitting position: detection by transesophageal echocardiography. Acta Anaesthesiol Scand 1998; 42: 643-7.

5. Bedell EA, Berge KH, Losasso TJ. Paradoxic air embolism during venous air embolism: transesophageal echocardiographic evidence of transpulmonary air passage. Anesthesiology 1994; 80: 947-50.

6. Tommasino C, Rizzardi R, Beretta L, Venturino M, Piccoli S. Cerebral ischemia after venous air embolism in the absence of intracardiac defects. J Neurosurg Anesthesiol 1996; 8: 30-4.

7. Butler BD, Hills BA. The lung as a filter for microbubbles. J Appl Physiol 1979; 47: 537-43.

8. Krowka MJ, Tajik AJ, Dikson ER, Wiesmer RH, Cortese DA. Intrapulmonary vascular dilatations (IPVD) in liver transplant candidates. Screening by two-dimensional contrast-enhanced echocardiography. Chest 1990; 97: 1165-70.

9. Hopkins WE, Waggoner AD, Barzilai B. Frequency and significance of intrapulmonary right-to-left shunting in end-stage hepatic disease. Am J Cardiol 1992; 70: 516-9.
10. Lee SY, Choi BIW, Kim JS, Park KS. Paradoxical air embolism during hepatic resection. Br J Anaesth 2002; 88: 136-8.

11. Nakayama R, Yano T, Mizutamari E, Ushijima K, Terasaki H. Possible pulmonary gas embolism associated with localized thermal therapy of the liver. Anesthesiology 2003; 99: 227-8.

12. Takatsuki M, Eguchi S, Yamanouchi K, Tokai H, Hidaka M, Soyama A, et al. Two-surgeon technique using saline-linked electric cautery and ultrasonic surgical aspirator in living donor hepatectomy: its safety and efficacy. Am J Surg 2009; 197: e25-7.

13. El Moghazy WM, Hedaya MS, Kaido T, Egawa H, Uemoto S. Takada Y. Two different methods for donor hepatic transection: cavitron ultrasonic surgical aspirator with bipolar cautery versus cavitron ultrasonic surgical aspirator with radiofrequency coagulator-A randomized controlled trial. Liver Transpl 2009; 15: 102-5.

14. Topal B, Aerts R, Peninckx F. Laparoscopic intrahepatic Glissonian approach for right hepatectomy is safe, simple, and reproducible. Surg Endosc 2007; 21: 2111. 